\title{
On First Appearance of Prime Differences
}

\author{
By L. J. Lander and T. R. Parkin
}

There has long been interest in strings of consecutive composite numbers appearing among the natural numbers. Most elementary texts on number theory include a discussion of how arbitrarily large gaps between consecutive primes can be constructed, for example [1]. Such constructive techniques lead to rather large numbers, however, and lower occurrences have been studied [2], [3] to gain insight into the subject.

In 1961, Gruenberger and Armerding examined the first six million primes (up to $P=104,395,289)[4]$ on a computer and produced certain statistics covering these primes [5]. They tabulated the primes forming the lower boundary for the first appearance of prime differences of prescribed lengths, where all intervening numbers are composite, up to the limit of the primes list. The largest difference found between two consecutive primes was 220 , and the smallest difference whose first appearance was not found was 186 .

An algorithm for direct search for prime-differences (usable on a computer of limited storage capacity) proceeds as follows:

(a) Start at a known prime, say $P_{a}$, below which all differences of interest are known.

(b) Form $P_{a}+D$, where $D$ is the smallest difference whose first appearance is unknown.

(c) From the point $P_{a}+D$, test the successively smaller numbers for primality by trial division or other technique until a prime $P_{b}$ is found.

(d) If $P_{b}>P_{a}$, replace $P_{a}$ by $P_{b}$ and repeat the algorithm.

(e) If $P_{b}=P_{a}$, start testing at $P_{a}+D$, and proceed to successively larger numbers until a prime $P_{c}$ is reached. $P_{c}-P_{a}$ is then a difference $\geq D$ between successive primes, and is recorded, unless such a difference has already occurred.

(f) Update $D$, if necessary, to the next larger difference whose first appearance is unknown; replace $P_{a}$ by $P_{c}$, and repeat the algorithm.

A computer program for the CDC 3200 was written to implement this algorithm, and Table I through the range $0<P<1.46 \times 10^{9}$ represents the data obtained from this program.

The algorithm itself guarantees that no difference of interest (i.e., $\geq$ smallest difference whose first appearance is unknown) will escape notice, while a separate check was run on the data in Table I. This check took the form of another computer program which read the Table I data as input, established the primality of $P_{a}$ and $P_{b}$ by testing for divisibility by primes up to the square root of $P_{a}$ or $P_{b}$, and explicitly exhibited all the prime factors of each odd number between the two primes. Thus, the differences listed are verified to be exactly as long as stated. Since all previous results in [5] were exactly duplicated (items of Table I for $D$ $\leq 184$ and $D=196,198,210,220$ ), the data may be regarded as accurate.

Received October 5, 1966. 
The primality testing process was designed to operate without using an extensive table of primes, while, at the same time, being made as rapid as practicable. First, the numbers to be tested were required to be prime to 210 . Since a sequence of consecutive numbers was being tested, a single division by 210 followed by a table lookup in a table of 210 positions sufficed to exclude all numbers not prime to 210 . Each entry of the table actually pointed to the next eligible number to be tested. Secondly, division by a few small primes was used. Since the range of interest quickly exceeded the single-precision word length of the computer (24 bits), the 48-bit hardware arithmetic of the machine was used. However, in order to avoid double-precision division as long as possible, the numbers being tested were reduced to single precision by subtraction of self-adjusting multiples of groups of small primes prior to division by those primes. The final step was division by all odd numbers prime to 6 (by alternately adding 2 , then 4 , to an appropriate starting prime) and less than the square root of the number tested, in order to verify the primality of the end points for the algorithm, and to determine that intervening numbers were composite. Of course, as soon as any eligible number being tested was found to be composite, it was rejected, and the next eligible number was selected for testing. Since there was no room in the program to store a table of pseudoprimes to the base 2, experiments with the converse of Fermat's Theorem to detect composite numbers were dropped when it was noted that the program spent the majority of its running time verifying the primality of the end points, rather than eliminating composite numbers between the end points.

With the availability of a larger computer memory in which to store a table of primes and their starting points with respect to a fixed field of bits, it becomes feasible to use a sieve technique for extending this search. However, with a very limited computer memory, the algorithm given above has the advantage of requiring only a table of previously found differences, and a starting point for each run, and thus could be used as a small background problem.

A program for the CDC 6600 was written to implement a sieve technique for generating and examining gaps in primes. This program occupied considerably more memory but ran significantly faster (partially due to an increase in computer speed) than the program described above. The sieve program allocated a block of computer memory in which consecutive bits represented the successive odd integers. A table of the first ten thousand primes was generated and stored by the program during initialization. Another table of starting points (i.e., index of the first bit in the field corresponding to a multiple of each prime in the stored table) for marking by each prime in the sieve field was also generated and saved. The program then cycled through successive bit fields marking bits corresponding to the odd composite numbers, then searched the field for gaps of interest. End effects at the boundaries of the sieve fields were noted so that gaps of interest would not be missed. Table I for $1.46 \times 10^{9}<P<1.096 \times 10^{10}$ presents the results obtained from this program.

In private correspondence Daniel Shanks suggested the possibility of extending Table I in [2] over the new differences found. Accordingly, Table I shows $\log P_{b} /(D-1)^{1 / 2}$, with each maximal gap $D$ marked with an asterisk. Maximal gaps, according to Shanks, are those larger than any preceding gap in the sequence of 
TABLE I

\begin{tabular}{|c|c|c|c|}
\hline$D$ & $P_{a}$ & $P_{b}$ & $\log P_{b} /(D-1)^{1 / 2}$ \\
\hline $2^{*}$ & 3 & 5 & 1.609 \\
\hline $4^{*}$ & 7 & 11 & 1.384 \\
\hline $6^{*}$ & 23 & 29 & 1.506 \\
\hline $8^{*}$ & 89 & 97 & 1.729 \\
\hline 10 & 139 & 149 & 1.668 \\
\hline 12 & 199 & 211 & 1.614 \\
\hline $14^{*}$ & 113 & 127 & 1.344 \\
\hline 16 & 1831 & 1847 & 1.942 \\
\hline $18^{*}$ & 523 & 541 & 1.526 \\
\hline $20^{*}$ & 887 & 907 & 1.562 \\
\hline $22^{*}$ & 1129 & 1151 & 1.538 \\
\hline 24 & 1669 & 1693 & 1.550 \\
\hline 26 & 2477 & 2503 & 1.565 \\
\hline 28 & 2971 & 2999 & 1.541 \\
\hline 30 & 4297 & 4327 & 1.555 \\
\hline 32 & 5591 & 5623 & 1.551 \\
\hline $34^{*}$ & 1327 & 1361 & 1.256 \\
\hline $36^{*}$ & 9551 & 9587 & 1.550 \\
\hline 38 & 30593 & 30631 & 1.698 \\
\hline 40 & 19333 & 19373 & 1.581 \\
\hline 42 & 16141 & 16183 & 1.514 \\
\hline $44^{*}$ & 15683 & 15727 & 1.474 \\
\hline 46 & 81463 & 81509 & 1.686 \\
\hline 48 & 28229 & 28277 & 1.495 \\
\hline 50 & 31907 & 31957 & 1.482 \\
\hline $52^{*}$ & 19609 & 19661 & 1.384 \\
\hline 54 & 35617 & 35671 & 1.440 \\
\hline 56 & 82073 & 82129 & 1.526 \\
\hline 58 & 44293 & 44351 & 1.417 \\
\hline 60 & 43331 & 43391 & 1.390 \\
\hline 62 & 34061 & 34123 & 1.336 \\
\hline 64 & 89689 & 89753 & 1.437 \\
\hline 66 & 162143 & 162209 & 1.488 \\
\hline 68 & 134513 & 134581 & 1.443 \\
\hline 70 & 173359 & 173429 & 1.452 \\
\hline $72^{*}$ & 31397 & 31469 & 1.229 \\
\hline 74 & 404597 & 404671 & 1.511 \\
\hline 76 & 212701 & 212777 & 1.417 \\
\hline 78 & 188029 & 188107 & 1.384 \\
\hline 80 & 542603 & 542683 & 1.486 \\
\hline 82 & 265621 & 265703 & 1.388 \\
\hline 84 & 461717 & 461801 & 1.432 \\
\hline $86^{*}$ & 155921 & 156007 & 1.297 \\
\hline 88 & 544279 & 544367 & 1.416 \\
\hline 90 & 404851 & 404941 & 1.369 \\
\hline 92 & 927869 & 927961 & 1.440 \\
\hline 94 & 1100977 & 1101071 & 1.443 \\
\hline $96^{*}$ & 360653 & 360749 & 1.313 \\
\hline 98 & 604073 & 604171 & 1.352 \\
\hline 100 & 396733 & 396833 & 1.296 \\
\hline
\end{tabular}


TABLE I-Continued

\begin{tabular}{|c|c|c|c|}
\hline$D$ & $P_{a}$ & $P_{b}$ & $\log P_{b} /(D-1)^{1 / 2}$ \\
\hline 102 & 1444309 & 1444411 & 1.411 \\
\hline 104 & 1388483 & 1388587 & 1.394 \\
\hline 106 & 1098847 & 1098953 & 1.357 \\
\hline 108 & 2238823 & 2238931 & 1.414 \\
\hline 110 & 1468277 & 1468387 & 1.360 \\
\hline $112^{*}$ & 370261 & 370373 & 1.217 \\
\hline $114^{*}$ & 492113 & 492227 & 1.233 \\
\hline 116 & 5845193 & 5845309 & 1.453 \\
\hline $118^{*}$ & 1349533 & 1349651 & 1.305 \\
\hline 120 & 1895359 & 1895479 & 1.325 \\
\hline 122 & 3117299 & 3117421 & 1.359 \\
\hline 124 & 6752623 & 6752747 & 1.418 \\
\hline 126 & 1671781 & 1671907 & 1.282 \\
\hline 128 & 3851459 & 3851587 & 1.346 \\
\hline 130 & 5518687 & 5518817 & 1.367 \\
\hline $132^{*}$ & 1357201 & 1357333 & 1.234 \\
\hline 134 & 6958667 & 6958801 & 1.366 \\
\hline 136 & 6371401 & 6371537 & 1.348 \\
\hline 138 & 3826019 & 3826157 & 1.295 \\
\hline 140 & 7621259 & 7621399 & 1.344 \\
\hline 142 & 10343761 & 10343903 & 1.360 \\
\hline 144 & 11981443 & 11981587 & 1.363 \\
\hline 146 & 6034247 & 6034393 & 1.297 \\
\hline $148^{*}$ & 2010733 & 2010881 & 1.197 \\
\hline 150 & 13626257 & 13626407 & 1.346 \\
\hline 152 & 8421251 & 8421403 & 1.298 \\
\hline $154^{*}$ & 4652353 & 4652507 & 1.241 \\
\hline 156 & 17983717 & 17983873 & 1.342 \\
\hline 158 & 49269581 & 49269739 & 1.414 \\
\hline 160 & 33803689 & 33803849 & 1.375 \\
\hline 162 & 39175217 & 39175379 & 1.378 \\
\hline 164 & 20285099 & 20285263 & 1.318 \\
\hline 166 & 83751121 & 83751287 & 1.420 \\
\hline 168 & 37305713 & 37305881 & 1.349 \\
\hline 170 & 27915737 & 27915907 & 1.319 \\
\hline 172 & 38394127 & 38394299 & 1.335 \\
\hline 174 & 52721113 & 52721287 & 1.352 \\
\hline 176 & 38089277 & 38089453 & 1.320 \\
\hline 178 & 39389989 & 39390167 & 1.315 \\
\hline $180^{*}$ & 17051707 & 17051887 & 1.245 \\
\hline 182 & 36271601 & 36271783 & 1.294 \\
\hline 184 & 79167733 & 79167917 & 1.344 \\
\hline 186 & 147684137 & 147684323 & 1.383 \\
\hline 188 & 134065829 & 134066017 & 1.368 \\
\hline 190 & 142414669 & 142414859 & 1.366 \\
\hline 192 & 123454691 & 123454883 & 1.348 \\
\hline 194 & 166726367 & 166726561 & 1.363 \\
\hline 196 & 70396393 & 70396589 & 1.294 \\
\hline 198 & 46006769 & 46006967 & 1.257 \\
\hline 200 & 378043979 & 378044179 & 1.400 \\
\hline
\end{tabular}


TABLE I-Continued

\begin{tabular}{|c|c|c|c|}
\hline$D$ & $P_{a}$ & $P_{b}$ & $\log P_{b} /(D-1)^{1 / 2}$ \\
\hline 202 & 107534587 & 107534789 & 1.304 \\
\hline 204 & 112098817 & 112099021 & 1.301 \\
\hline 206 & 232423823 & 232424029 & 1.345 \\
\hline 208 & 192983851 & 192984059 & 1.326 \\
\hline $210^{*}$ & 20831323 & 20831533 & 1.166 \\
\hline 212 & 215949407 & 215949619 & 1.321 \\
\hline 214 & 253878403 & 253878617 & 1.326 \\
\hline 216 & 202551667 & 202551883 & 1.304 \\
\hline 218 & 327966101 & 327966319 & 1.331 \\
\hline $220^{*}$ & 47326693 & 47326913 & 1.194 \\
\hline $222^{*}$ & 122164747 & 122164969 & 1.253 \\
\hline 224 & 409866323 & 409866547 & 1.328 \\
\hline 226 & 519653371 & 519653597 & 1.338 \\
\hline 228 & 895858039 & 895858267 & 1.368 \\
\hline 230 & 607010093 & 607010323 & 1.336 \\
\hline 232 & 525436489 & 525436721 & 1.321 \\
\hline $234^{*}$ & 189695659 & 189695893 & 1.249 \\
\hline 236 & 216668603 & 216668839 & 1.252 \\
\hline 238 & 673919143 & 673919381 & 1.320 \\
\hline 240 & 391995431 & 391995671 & 1.280 \\
\hline 242 & 367876529 & 367876771 & 1.270 \\
\hline 244 & 693103639 & 693103883 & 1.306 \\
\hline 246 & 555142061 & 555142307 & 1.286 \\
\hline $248^{*}$ & 191912783 & 191913031 & 1.214 \\
\hline $250^{*}$ & 387096133 & 387096383 & 1.253 \\
\hline 252 & 630045137 & 630045389 & 1.279 \\
\hline 254 & 1202442089 & 1202442343 & 1.314 \\
\hline 256 & 1872851947 & 1872852203 & 1.337 \\
\hline 258 & 1316355323 & 1316355581 & 1.310 \\
\hline 260 & 944192807 & 944193067 & 1.284 \\
\hline 262 & 1649328997 & 1649329259 & 1.314 \\
\hline 264 & 2357881993 & 2357882257 & 1.331 \\
\hline 266 & 1438779821 & 1438780087 & 1.295 \\
\hline 268 & 1579306789 & 1579307057 & 1.296 \\
\hline 270 & 1391048047 & 1391048317 & 1.284 \\
\hline 272 & 1851255191 & 1851255463 & 1.296 \\
\hline 274 & 1282463269 & 1282463543 & 1.269 \\
\hline 276 & 649580171 & 649580447 & 1.224 \\
\hline 278 & 4260928601 & 4260928879 & 1.332 \\
\hline 280 & 1855047163 & 1855047443 & 1.278 \\
\hline $282^{*}$ & 436273009 & 436273291 & 1.187 \\
\hline 284 & 1667186459 & 1667186743 & 1.262 \\
\hline 286 & 2842739311 & 2842739597 & 1.289 \\
\hline $288^{*}$ & 1294268491 & 1294268779 & 1.238 \\
\hline 290 & 1948819133 & 1948819423 & 1.258 \\
\hline $292^{*}$ & 1453168141 & 1453168433 & 1.237 \\
\hline 294 & 5692630189 & 5692630483 & 1.312 \\
\hline 296 & 5260030511 & 5260030807 & 1.303 \\
\hline 298 & 8650524583 & 8650524881 & 1.328 \\
\hline 300 & 4758958741 & 4758959041 & 1.289 \\
\hline
\end{tabular}


TABLE I-Continued

\begin{tabular}{l|r|r|c}
\hline$D$ & $P_{a}$ & \multicolumn{1}{|c}{$P_{b}$} & $\log P_{b} /(D-1)^{1 / 2}$ \\
\hline 302 & 6675573497 & 6675573799 & 1.304 \\
304 & 2433630109 & 2433630413 & 1.242 \\
306 & 3917587237 & 3917587543 & 1.265 \\
308 & 5490459101 & 5490459409 & 1.280 \\
310 & 4024713661 & 4024713971 & 1.258 \\
312 & 6570018347 & 6570018659 & 1.282 \\
314 & 8948418749 & 8948419063 & 1.295 \\
318 & 4372999721 & 4373000039 & 1.247 \\
$320^{*}$ & 2300942549 & 2300942869 & 1.207 \\
322 & 7961074441 & 7961074763 & 1.272 \\
324 & 10958687879 & 10958688203 & 1.286 \\
326 & 5837935373 & 5837935699 & 1.247 \\
330 & 6291356009 & 6291356339 & 1.244 \\
332 & 5893180121 & 5893180453 & 1.237 \\
$336^{*}$ & 3842610773 & 3842611109 & 1.206 \\
340 & 8605261447 & 8605261787 & 1.242 \\
$354^{*}$ & 4302407359 & 4302407713 & 1.181 \\
$382^{*}$ & 10726904659 & 10726905041 & 1.183 \\
\hline
\end{tabular}

primes. These data tend to support the conjectured relation in [2], namely that log $P_{b} \sim(D-1)^{1 / 2}$ for maximal gaps, and, also, possibly, for all gaps at the point of their first appearance. For example, $D=316$ is the first difference that does not appear in our table, but since $\log P_{b} /(D-1)^{1 / 2}$ is consistently $<4 / 3$ for $D>256$, it is not unreasonable to guess that $D=316$ will appear before $\exp (4(\sqrt{ } 315) / 3)$ $=10^{10 \cdot 277}$.

The authors wish to acknowledge the assistance of Miss Pauline Parkin who prepared the checking program mentioned above.

Aerospace Corporation

Los Angeles, California 90045

1. J. V. Uspensky \& M. A. Heaslet, Elementary Number Theory, McGraw-Hill, New York, 1939 , p. 90 , paragraph 14 . MR 1,38 .

2. Daniel Shanks, "On maximal gaps between successive primes," Math. Comp., v. 18, 1964, pp. 646-651. MR 29 \#4745.

3. S. M. Johnson, "An elementary remark on maximal gaps between successive primes," Math. Comp., v. 19, 1965, pp. 675-676.

4. C. L. BAker \& F. J. Gruenberger, The First Six Million Prime Numbers, The RAND Corp., July 1957, Microcard Foundation, West Salem, Wis., 1959.

5. F. Gruenberger \& G. Armerding, Statistics on the First Six Million Prime Numbers, Paper P-2460, The RAND Corp., Santa Monica, Calif., 1961. 